\title{
Design and Application of High-Speed Data Acquisition Aboard a High-Power Rocket in an Undergraduate Experimental Engineering Course
}

\section{Dr. Mary Cardenas, Harvey Mudd College}

Dr. Cardenas earned her B.Sc. in Aerospace Engineering from Iowa State Engineering. She joined Rocketdyne as a propulsion engineer and worked on the Space Shuttle Main Engines, Atlas Engine, and the X-30 propulsion system. Dr. Cardenas received her M.Sc. and Ph.D. in Environmental and Mechanical Engineering from the University of California, Santa Barbara, studying the transport and fate of PCBs and sediments in the Saginaw River. She has been a member of the Engineering department at Harvey Mudd College since 1995, and has served as Associate Dean of Faculty for Academic Affairs. She is the co-author of the Journal of Engineering Education paper, "Use of "Studio" Methods in the Introductory Engineering Design Curriculum" and co-developer of the sophomore-level rocket-based experimental engineering lab course at HMC. Dr. Cardenas is currently exploring novel pedagogy for Introductory Environmental Engineering courses and researching marine hydrokinetic turbines.

\section{Prof. R. Erik Spjut, Harvey Mudd College}

Prof. Spjut is the Union Oil Company Design Fellow and Professor of Engineering in the Department of Engineering at Harvey Mudd College. He has served a Director of the Engineering Clinic at Harvey Mudd and has been a Visiting Professor at Olin College Of Engineering, California State Polytechnic University, Pomona, and the California Institute Of Technology. He was also the John Chipman Assistant Professor of Chemical Process Metallurgy in the Department of Materials Science and Engineering at the Massachusetts Institute Of Technology. Prof. Spjut has taught most of the required engineering courses and has been involved in innovative pedagogy at Harvey Mudd. 


\title{
Design and Application of High-Speed Data Acquisition Aboard a High- Power Rocket in an Undergraduate Experimental Engineering Course
}

\begin{abstract}
Undergraduate students in the Experimental Engineering Course at Harvey Mudd College design and fly sensor and signal-conditioning packages on high-powered rockets (total impulse of between 100 Ns and 600 Ns.) The data from these flights are collected using data loggers; this paper will describe the various data loggers used over the history of the course, from commercial rocketry flight computers through our current custom-designed and -built data logger, and the impact on student learning and quality of experimental data. A main objective of the course is that students move through a sequence of choosing scientific goals for their mission; model the expected behavior of the flight; select, design, and build an appropriate sensor package to measure phenomena tied to the scientific mission of their flight; and compare expected behavior to their measured results from the flight.
\end{abstract}

In designing our flight data logger, we explicitly made choices regarding the ranking of various aspects of student learning. In particular, we wanted students to focus on proper signal conditioning for data logger input (voltages and impedance), and choice of number of channels and sample rate. A commercial 6-channel, 200 SPS per channel data logger was used (out of necessity) in the early offerings of the course, the use of which caused the students to struggle inordinately with choices involving small number of channels and low sampling rate. This affected the students' ability to achieve their scientific goals, especially the ability to measure different types of data for comparison purposes. As the course developed and we progressed through our data logger design, we chose 16 channels and a much-higher composite sampling rate of $400 \mathrm{kSPS}$ (ranging from $25 \mathrm{kSPS} / \mathrm{channel}$ for 16 channels, up to $200 \mathrm{kSPS} / \mathrm{channel}$ for 2 channels). This choice was informed by a typical mission to measure vehicle acceleration, velocity, position, orientation, and vibrational modes, which requires 12 to 15 channels, and a composite sampling rate greater than $320 \mathrm{kSPS}$. The custom data logger allows the students a better chance at acquiring good data to satisfy their mission goals.

Rubrics were used to assess four years of student work objectives relating to students' use of data acquisition systems and demonstration of experimental and analytical skills. Students using the MuddLog16 scored higher on the safe and proper use of data acquisition systems, and acquired more and better experimental data, which allowed them to satisfy their scientific mission. We saw no major differences in students' skills in scaling input voltages as we used different data loggers; students generally satisfied this objective no matter which data logger was used. Student consideration of buffering inputs was improved when we moved to the MuddLog 16. The increase in number of channels and the higher sampling rate had the not-surprising effect of improving the quality of the experimental data acquired; we saw improvements in the ability of 
the students to more-completely compare experimental results to analytical or simulated predictions, satisfying a major learning objective. The higher sampling rate of the MuddLog16 had the effect of allowing students to be less-attentive to the potential of aliasing; future versions of the course should examine means to ensure students acquire and understand aliased data.

\section{Introduction}

Experimental Engineering at Harvey Mudd College is an intense multi-faceted sophomore-level, semester-long course. The stated learning objectives for the course are:

1. Demonstrate hardware and equipment skills:

a. Demonstrate the safe and proper use of basic laboratory equipment: e.g., digital multimeter (DMM), signal generator, oscilloscope, breadboard, and analog transducers.

b. Demonstrate the safe and proper use of computer-based and embedded-processorbased data acquisition systems.

c. Demonstrate proper techniques for debugging/troubleshooting an experimental setup.

d. Design, build, and fly a custom set of transducers to make engineering and/or scientific measurements.

2. Demonstrate experimental and analytical skills:

a. Demonstrate the design/planning and completion of safe experiments to answer open-ended questions.

b. Demonstrate manipulation and presentation of experimentally-obtained data to answer open-ended questions.

c. Analyze and compare the results of mathematical and computer modeling of an experiment with actual experimental results.

3. Demonstrate the beginnings of professional practice:

a. Effectively communicate in written form the design, completion, and analysis of experiments to answer open-ended questions.

b. Effectively communicate by oral presentation and Q-and-A session the design, completion, and analysis of experiments to answer open-ended questions.

We have assessed these objectives with both direct and indirect measures since the beginning of the course. A full discussion of the objectives, assessment, and changes to the course far exceeds the scope of this paper. For this paper we are focused on Objectives $1 \mathrm{~b}$ (data acquisition), 2a (design \& complete experiments), and 2c (compare modeling with experiment), and on the design objectives of the data acquisition system and their effects on student learning. In particular, we discuss the data logger used for the final student field project, its history, design objectives, and the effects on student outcomes. 


\section{Background}

In experimental work, it is important to record and store measurements. In the past, this has been done by manually reading measurement values from various instrumentation. These values, and the times at which the data were taken, were recorded in ink in a paper laboratory notebook. Rough plots of data were often sketched by hand in these notebooks, or were mechanically plotted using strip chart recorders. The advent of the microcontroller led to sampling and digitizing of signals from measurement sensors, and computerized storage of these data. As microcontrollers became increasingly powerful and less expensive, computerized data loggers and data acquisition systems became common ways to record and store experimental data.

Broadly defined, a data acquisition system (DAQ) consists of sensors, circuitry to modify/condition the sensor signal, analog-to-digital converters, and either on-board storage, or a means to interface with a computer for remote storage of data. Modern DAQ, especially PCbased systems, provide computation abilities and graphical interfaces to quickly process and visualize data. A stand-alone (or traditional) data logger is a type of DAQ that is not tethered to a $\mathrm{PC}$, and therefore has its own power source, and on-board storage of data (often done using a memory card.) Channel count and sample rates are some important characteristics of data loggers.

The teaching of data acquisition principles in undergraduate engineering is important because computer-based data acquisition and control is ubiquitous in industrial and laboratory contexts. Data measurements are taken to characterize and analyze performance of a device or system; validate analytical models; further understanding of physical phenomena; monitor and control performance of manufacturing, automation, or processes; and monitor equipment. Dataacquisition education can be tied to principles of signal processing and electrical engineering, and reinforce graphing skills and presentation.

Ray $^{1,2}$ described a sophomore-level instrumentation and data-acquisition course at Bloomsburg University. The course was offered to electronics engineering technology students. The first twothirds of the course focused on lab work related to concepts of data acquisition and control, and the last third of the course involved student-initiated projects, such as DC motor drive systems; liquid-level control; and an environmental automation system controlling temperature and lighting inside an enclosure. The data acquisition system included a DAQ board tethered to a PC, with a controller and conditioning accessory, as well as various sensors depending on the project. Ray reported students recommended having more time allotted for the final project, and enjoyed being able to define their own project. He noted that students had little previous experience in testing and debugging systems, and generally did not break their work into testable sub-modules during debugging. 
The Geosciences department at Texas Tech University describes three graduate level courses in meteorological data acquisition and instrumentation ${ }^{3}$. In the first course, student teams work with a PC-based DAQ, LabVIEW Virtual Instrument Workbench, and meteorological sensors to learn how to build and use data acquisition systems. They also are provided with Campbell Scientific data loggers in order to learn their use, and how to develop acquisition software. The students in these courses were from multiple departments, including engineering students. Of note is their observation that while 19\% of survey respondents report arriving to graduate school 'at TTU with some experience with sensors, only $5 \%$ of respondents understand how to interface with them.' The third course in the TTU sequence involves a field experience to research kinematic and thermodynamic gradients within supercells of thunderstorms. The atmospheric science faculty noted that students who have completed these courses had dramatically increased skills 'to complete more independent, observationally based research projects.'

Mason $^{4}$ developed a handheld DAQ from a PocketPC and a low-cost DAQ card for use in an undergraduate (junior-level) mechanical engineering laboratory course at Seattle University. The portable DAQ replaced desktop DAQ systems that had been in use. The lab experiments in the course were designed so that students built on previous learning (and coding) with the idea that students would re-use their work in future experiments. The handheld DAQ was found to be a good replacement for the desktop systems, with, of course, the lower sampling rate of the handheld system being of note. However, Mason did not believe this was a serious issue, since students rarely took data using high sampling frequencies. The handheld DAQ was also used to acquire data in a thermodynamics lab course, as well as in a capstone design course.

Warren et al. ${ }^{5}$ developed RASCL (Rapid Analysis and Signal Conditioning Laboratory), a hardware bridge for the myDAQ, and the hardware was used in an Instrumentation course at Kansas State University. The lab introduced students to instrumentation amplifiers in order to acquire data from biomedical electrodes using RASCL. The authors noted that, although they hoped that students would use these tools using personal laptops outside of class time, most students only worked on lab computers during lab time. In particular, students did not build and test circuitry outside of lab time. However, the authors reported that learning objectives had all been met, and that students found the hardware tool useful for data acquisition.

During development of the E80 course, we moved from a pre-selected set of sensors (when the commercial data logger was used) towards a version more in line with Ray after the custom logger was implemented. In the current version of E80, the students define their own scientific goals and sensor packages for the final project; the custom 16-channel data logger with the higher sampling rate allowed the more open-ended choice by the students. Based on student course evaluations, we noted similar changes in student satisfaction and sense of ownership in the final project. Instructors in the E80 course also recognized the same kinds of debugging issues as reported in Ray, and in response, have encouraged the students to break up their debugging into examining sub-modules. Similar to Mason's work, we designed the first half of 
E80 so that students would learn the basic circuits with the idea that students would re-use their work in the final project. Although we have yet not performed formal assessment measuring how much students re-use their work in our capstone course, anecdotal evidence suggests that our junior and senior Clinic students have increased their skills and familiarity in the selection and use of sensors, and in acquiring data using PC-based DAQ systems. The custom data logger is more representative of data acquisition systems that are used in Clinic (and industry), and model calibration using multiple channels of experimental data is a common task in our capstone course. Regarding Warren's observation that students did not necessarily use time outside of lab to build and test, our structure of E80 was designed such that students felt restricted from doing a lot of work with hardware outside the lab during the first half of the course, but were released from those restrictions for the second half of the course when each team received their own custom data logger. Students were allowed to populate their PC boards and test their data loggers both during lab, and outside lab time. Although work outside lab time is good, we found it necessary, over the development of the course, to set definite progress checks for the students, especially during the weeks leading up to flight, in order to curtail the "we can build it in one night" mindset.

\section{Description of E80 course}

Experimental Engineering at Harvey Mudd College is a sophomore-level, semester-long course, involving multiple experiments covering a number of engineering disciplines. The objectives of the course as noted in the introduction are to teach basic instrumentation and measurement techniques; good lab notebook practice; technical report writing; analysis and presentation of data; the usage of experimental results for engineering design purposes; and the beginnings of professional practice.

The course explicitly requires learning in multiple disciplines but directs all of the experiments to a final goal: to build, instrument, and fly a small rocket; and analyze and report on the data collected during the flight. The course walks the students through modeling of the rocket performance based on weight, vibration, strength, drag, and engine test data; and the implementation and configuration of an instrument package and data acquisition system. The students have various objectives and constraints related to their scientific goals and project budget; therefore they are required to choose from among alternatives when designing their sensor package. Each student team builds and instruments a rocket, and test flights are made where the students collect experimental data. If weather conditions and the state of the vehicle permit (i.e., the rocket wasn't damaged or destroyed during flight or recovery), each student team may get data from up to four flights.

The course format consists of two large lectures, and two three-hour laboratory sessions per week. Course enrollment over the past five years has ranged from approximately 60 to 80 students per semester. These 60-80 students are divided into four sections of up to 20 students. 
The typical staffing for the course is one professor per 20 students. While this faculty-student ratio is considerably higher than that of most engineering programs, it is consistent with Harvey Mudd's approach to undergraduate education. The students are placed in teams of four students, and perform their laboratory work as teams.

The laboratory experiments in the course span various engineering disciplines. Electrical engineering and electronics is emphasized, since modern instrumentation and data acquisition relies heavily on those disciplines. The students learn basic electrical measurements and design/test an op-amp-based low-pass (anti-aliasing) filter along with other signal-conditioning circuits. This filter can be used during the data acquisition phase of the launch. In order to prepare the students for the various instrumentation tasks, there are laboratories focusing on data acquisition (pressure, temperature, acceleration, and rotation-rate measurements) and the use of modern computer-based data-acquisition systems such as LabVIEW along with the myDAQ device. Mechanical and aerospace engineering topics are also fundamental to rocket flight; in particular, fluid mechanics and trajectory modeling are important. In order to develop the students' understanding of wind tunnel measurements, there is a lab involving drag measurements and calculations for standard shapes and the model rocket. The students also build on their introductory physics knowledge to model vehicle kinetics and flight trajectory, and also perform static engine tests on the model rocket motors to measure the thrust curve.

\section{Data logger objectives and design goals}

One of the principal goals of E80 is for the students to complete the sequence of: deciding on a mission for their rocket; modeling the expected behavior of the flight; selecting, designing, and building the sensor package to measure the mission goals; and comparing the expected behavior from the modeling with the measured results from the flight. How this sequence gets mapped to low-level skills is one of the principal issues that we had to wrestle with while designing the course and the hardware. Since one can't test for every possible mix of low level skills, the instructors needed to make some decisions. The goal we decided upon for the rocket-mounted data logger was to provide the students a (relatively) easy-to-use and reliable way to store their data so they could focus on their sensors and not data-storage issues. We deliberately did not want the students to wrestle with hardware limited sampling rate or bit depth. While both are important concepts, given that the students design, build, test, and fly a complete instrumentation and data acquisition system in four weeks, we felt that unavoidable hardware aliasing and bit depth were topics that we didn't want to require the students to deal with. Consequently, we had a list of low-level objectives for which the students would be responsible ("The Student List" Table 1) and ones that the data loggers would take care of behind the scenes ("The Behind-theScenes" list - Table 2). 
Table 1. Low-level objectives that the students are responsible for

\section{The Student List}

Scale the input voltages to the proper input range for the data logger

Condition the signal to account for the data logger input impedance

Choose the number of channels

Choose the sample rate

Provide power to the data logger from a battery

Table 2. Low-level objectives that the data logger will take care of behind the scenes

The Behind-The-Scenes List

Provide enough channels so that a student can fully characterize the desired phenomena

Keep quantization noise to a minimum even if the student doesn't get signal scaling exactly

right

Provide sufficient dynamic range for wide-dynamic-range signal such as strain gauges, vibration sensors, accelerometers, and photodiodes

Provide a 10-times-Nyquist sampling rate for the fastest sensors so the hardware anti-aliasing can be fairly gentle or missing

Provide enough storage so that students aren't stressing over how long the prep-launch-recover cycle takes

Protect against damage to the logger caused by either power polarity reversal or signal overvoltage

Two typical performance missions that students often decide on in E80 are determining the acceleration, velocity, position, and orientation of the rocket during flight, and measuring the vibrational modes during flight. The sensor count for these two missions combined is seven for the 7-DOF IMU, one for an altimeter, one for a Pitot tube, and three-to-six vibration sensors, for a total of twelve to fifteen sensors. Consequently, the design goal was for sixteen input channels. The highest observable vibrational mode in the rockets is typically around $1000 \mathrm{~Hz}$, requiring a minimum sampling rate of $2000 \mathrm{~Hz}$. With a design goal of $10 \times$ the minimum sampling frequency, a sampling rate of $20 \mathrm{kSPS}$ per channel or a composite of $320 \mathrm{kSPS}$ was desired. The dynamic range and quantization goals could be satisfied with 16-bit ADCs. At the time of the course development in 2007, a commercial 16-channel, 16-bit datalogger with a composite 320 kSPS was both far too expensive and far too large to be practical.

\section{Data logger history and description}

The three data loggers used in E80 will be described. These are the R-DAS (Rocket DataAcquisition System), a commercial system used in initial offerings of the course; the Logomatic (a less-expensive, more-flexible commercial system used next); and the MuddLog16, our custom design used from 2010 onwards. Table 3 summarizes the three data loggers. 
Table 3. Comparison of Data Logger Specifications

\begin{tabular}{|l|r|r|r|}
\hline & R-DAS Tiny & Logomatic & \multicolumn{1}{|c|}{ MuddLog16 } \\
\hline Source & AED Electronics & Sparkfun & In-House \\
\hline Channels & $2+6$ & 8 & 16 \\
\hline Input Range & 0 -to-5 V & 0 -to-3 V & 0 -to-3.3 V \\
\hline Max SR per channel & $200 \mathrm{SPS}$ & $1500 \mathrm{SPS}$ & $200 \mathrm{kSPS}$ \\
\hline Max composite SR & $1600 \mathrm{SPS}$ & $1500 \mathrm{SPS}$ & $400 \mathrm{kSPS}$ \\
\hline Bits & 10 & 10 & 16 \\
\hline Dynamic Range & $60 \mathrm{~dB}$ & $60 \mathrm{~dB}$ & $96 \mathrm{~dB}$ \\
\hline Input Impedance & $50 \mathrm{k} \Omega$ & $20 \mathrm{k} \Omega$ & $2.2 \mathrm{k} \Omega$ \\
\hline Storage Location & internal & microSD & microSD \\
\hline Max storage & $1.6 \mathrm{MB}$ & $4 \mathrm{~GB}$ & $16 \mathrm{~GB}$ \\
\hline Logging Time (typical) & 8 minutes & 300 hours & 35 hours \\
\hline Years in Use & $2008-2009$ & $2010-2012$ & $2013-2016$ \\
\hline Other & $10 \mathrm{~Hz} \mathrm{Telemetry}$ & $\begin{array}{r}\text { Binary or ASCII } \\
\text { file format }\end{array}$ & $\begin{array}{r}\text { Only Binary file } \\
\text { format }\end{array}$ \\
\hline
\end{tabular}

The R-DAS, the closest practical commercially-available system, was used for the budgetconstrained first and second years $(2008,2009)$. It had a $900 \mathrm{MHz}$ telemetry unit ${ }^{6}$ that integrated with the R-DAS Tiny ${ }^{7}$ (Figure 1.) Telemetry was 8 channels of sampled analog data at 10 SPS and 10-bit resolution. It also ostensibly transmits four channels of digital data and one channel of GPS data (if you use AED's GPS and can get it to lock. We never could). The R-DAS allows 8 analog channels at a sampling rate of $200 \mathrm{~Hz}$. The R-DAS used two of the 8 analog channels to store data from a pressure sensor for altitude and a single-axis $\pm 50 \mathrm{~g}$ accelerometer. The students had to choose which set of sensors to measure on the remaining six channels: six vibration sensors, two pressure sensors and four thermistors, or a six-axis IMU. This sampling rate, when used to measure vibration data to determine rocket-body modal frequencies, meant that aliasing of the 1st, 2nd, and 3rd mode frequencies occurred. The relatively coarse 10-bit ADC lead to large quantization artifacts in the data.

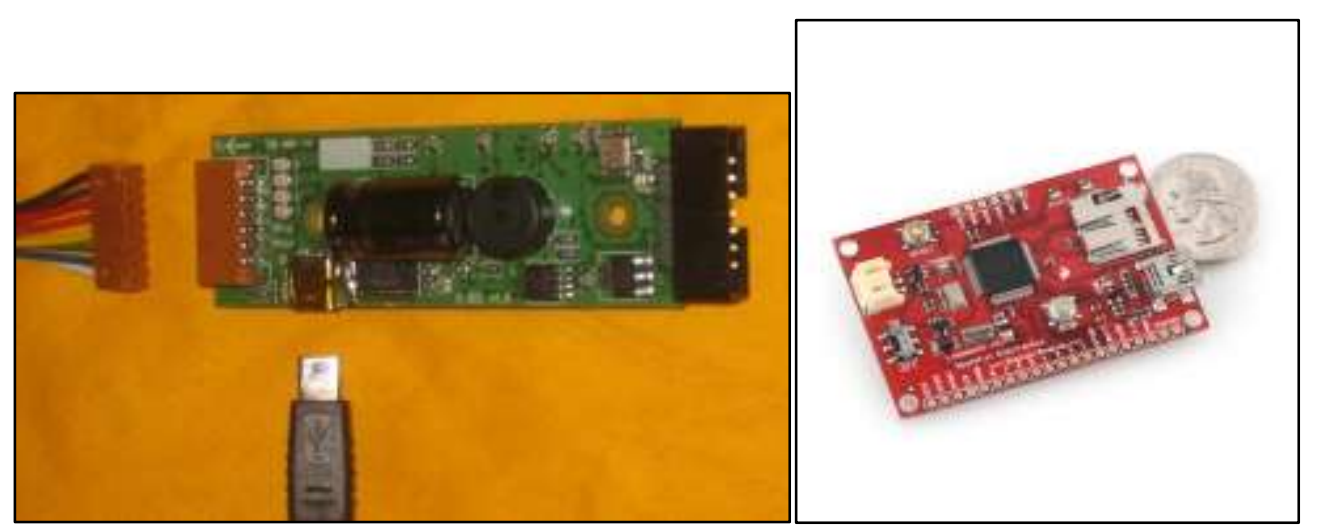

Figure 1. R-DAS ${ }^{8}$

Figure 2. SparkFun Logomatic ${ }^{9}$ 
Starting in 2011, the SparkFun Logomatic (Figure 2), was substituted for the R-DAS at about $1 / 4$ of the cost. The Logomatic has 8 channels of 10-bit acquisition at 200 SPS per channel; essentially the same specifications as the R-DAS but with more flexibility for the students in choosing sensors and channels, and the data were stored on a microSD card, which made configuration and data processing much easier for the students.

Neither of these devices were acceptable from the channel-count, dynamic range, quantization noise, or sampling rate criteria, resulting in students having to pay much more attention to these issues on our behind-the-scene list than was desirable.

Despite its shortcomings, the SparkFun Logomatic had been fairly successful in terms of the students fitting it in the rocket payload section and routing signals to it, so its physical dimensions, microSD card, and general pin placement were used to guide the design of the MuddLog16 data logger (Figure 3). The data logger uses two Analog Devices AD7689 PulSAR ADCs. Each ADC has 8 channels and a maximum throughput of $250 \mathrm{kSPS}$, for a theoretical throughput of $500 \mathrm{kSPS}$ and a tested throughput of $400 \mathrm{kSPS}$. If all sixteen channels are used at the maximum rate, the sample rate is $25 \mathrm{kSPS}$ with a Nyquist frequency of $12.5 \mathrm{kHz}$ for each channel, which meets the 10-times goal. The ADCs have an input impedance of approximately $2200 \Omega$ requiring the students to buffer their transducers which was one of the intended student outcomes. The ADCs are 16-bit devices, with a specified dynamic range of over $90 \mathrm{~dB} .24$ bits would be even better, but given the current market, the 16 bits is sufficient to provide the students with a wide dynamic range without requiring them to squeeze the maximum range out of their signal conditioning. The data are written onto a $16 \mathrm{~GB}$ class 10 microSD card. We have provided LabVIEW VIs or MATLAB m-files for the students to read the data off of the microSD cards. The data logger is configured through a configuration file with options for the number of channels ( 2 through 16 in pairs) and composite sample rate. The on-board voltage regulators are protected against reverse polarity and will work for any input voltage from $6 \mathrm{~V}$ to $18 \mathrm{~V}$ (the students usually use a standard $9 \mathrm{~V}$ battery.) All 16 inputs are protected against overvoltage and reverse polarity with Schottky diodes. With the power and signal-input protection, and the 16 channels of 16-bit acquisition at a composite $400 \mathrm{kSPS}$ rate, the custom data logger has permitted us to largely achieve our behind-the-scenes list. Table 4 summarizes the degree to which each data logger met the design goals.

There are two slightly different versions of the logger: the MuddLog16 v $3^{10,11}$ from December 2012, and the MuddLog16 v4 ${ }^{12,13}$ from March 2015. They differ in the type and placement of the microSD holder and the as-manufactured pin height. In the 2013 and 2014 offerings of E80, MuddLog16 v3 was used exclusively. For 2015, a combination of v3 and v4 MuddLog16 were used. 
Table 4. Data Logger Design Goals Summary

\begin{tabular}{|c|c|c|c|}
\hline & R-DAS Tiny & Logomatic & MuddLog16 \\
\hline \multicolumn{4}{|l|}{$\begin{array}{l}\text { Design Goals (Behind the } \\
\text { Scenes List) }\end{array}$} \\
\hline Channel Count & $50 \%$ low & $50 \%$ low & met \\
\hline Bit Depth & 6 bits low & 6 bits low & met \\
\hline Dynamic Range & $30 \mathrm{~dB}$ low & $30 \mathrm{~dB}$ low & met \\
\hline Sample Rate & 100X low & $100 X$ low & met \\
\hline Storage Capacity & min. acceptable & far exceeds & far exceeds \\
\hline Input Protection & no & no & met \\
\hline \multicolumn{4}{|l|}{ Student Responsibility List } \\
\hline Scale voltage for input & acceptable & acceptable & acceptable \\
\hline Condition for impedance & largely ignored & largely ignored & acceptable \\
\hline Choose \# of channels & student frustration & student frustration & acceptable \\
\hline Choose sample rate & student frustration & student Frustration & acceptable \\
\hline Battery power & able & table & acceptable \\
\hline
\end{tabular}

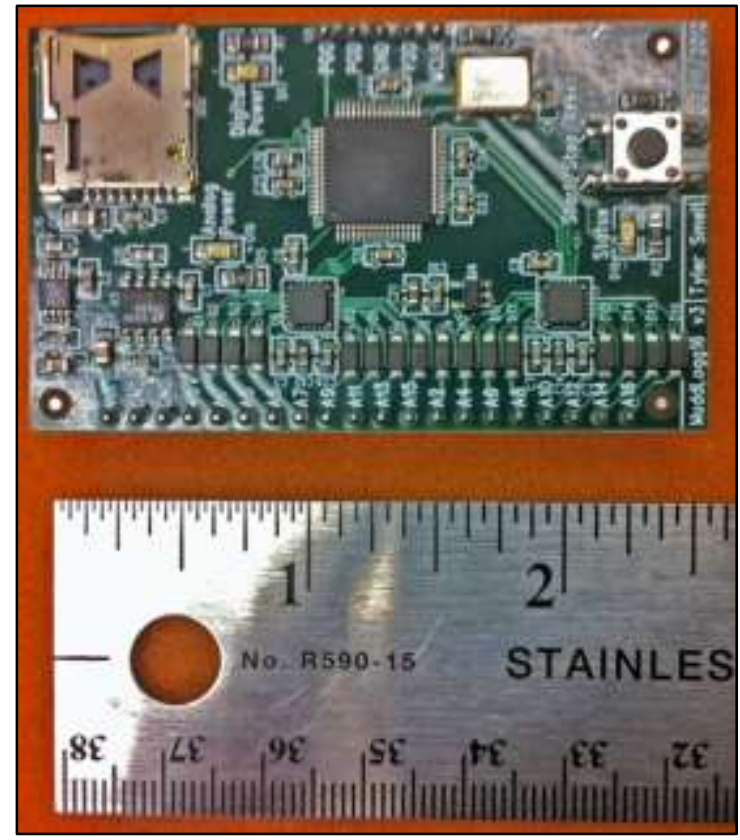

Figure 3. MuddLog16 v3

\section{Examples of student work}

Comparison of the data recorded and processed by the students in 2008 using the R-DAS with those from students in 2014 and 2015 using the new data logger shows the remarkable difference between the old data quality and the new data quality. The question of how the data-quality difference translates into differences in student learning outcomes is addressed in the Results section. 
Figures 4 and 5 from 2008 show a great deal of quantization staircasing and the poor altitude resolution. The third and fifth columns of plots in Figure 6 are the sensor data from the accelerometers and rate gyros from a 2008 flight. The plot in the third column on the third row is the built-in accelerometer on the R-DAS. It shows severe quantization noise. The other accelerometer and rate gyro plots show much less stairstepping due to careful attention to scaling the signals for the 10-bit ADC. The other plots on Figure 6 are axial position, velocity, and rotation angle calculated by numerically integrating the data. While the curves are smoother, the offset integration errors are unacceptably large. The flight reached an apogee around $300 \mathrm{~m}$, and travelled downrange about $250 \mathrm{~m}$. The integrated curves show axial endpoints of $-1000 \mathrm{~m}, 2000$ $\mathrm{m}$, and $9000 \mathrm{~m}$ respectively. The integration errors were mostly due to errors in determining correct offsets and the noise in the accelerometers. The R-DAS quantization errors were mostly minor contributors.

In comparison, Figures 7 and 8 show student data taken with the new data logger. There is no stairstepping quantization and the plots show fine details of the sensor output. The variations that appear like noise in the measured altitude versus time curve in Figure 7 are actual pressure variations from the airflow past the rocket during descent. Likewise, in Figure 8, the variations in the temperature curves are actual variations in the sensors and processing circuits and not artifacts in the digitization.

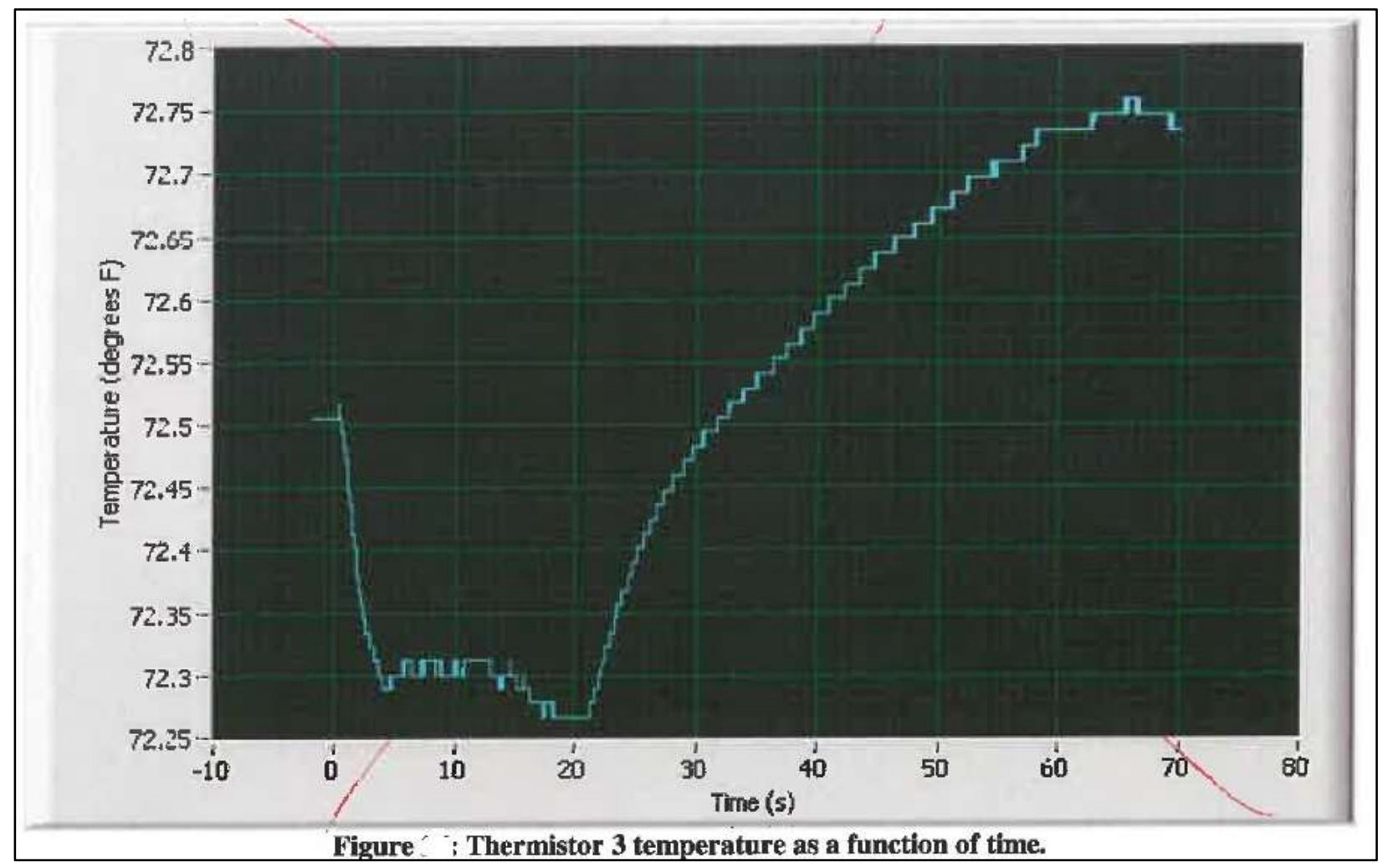

Figure 4. Flight data from a thermistor acquired using the R-DAS tiny in 2008 


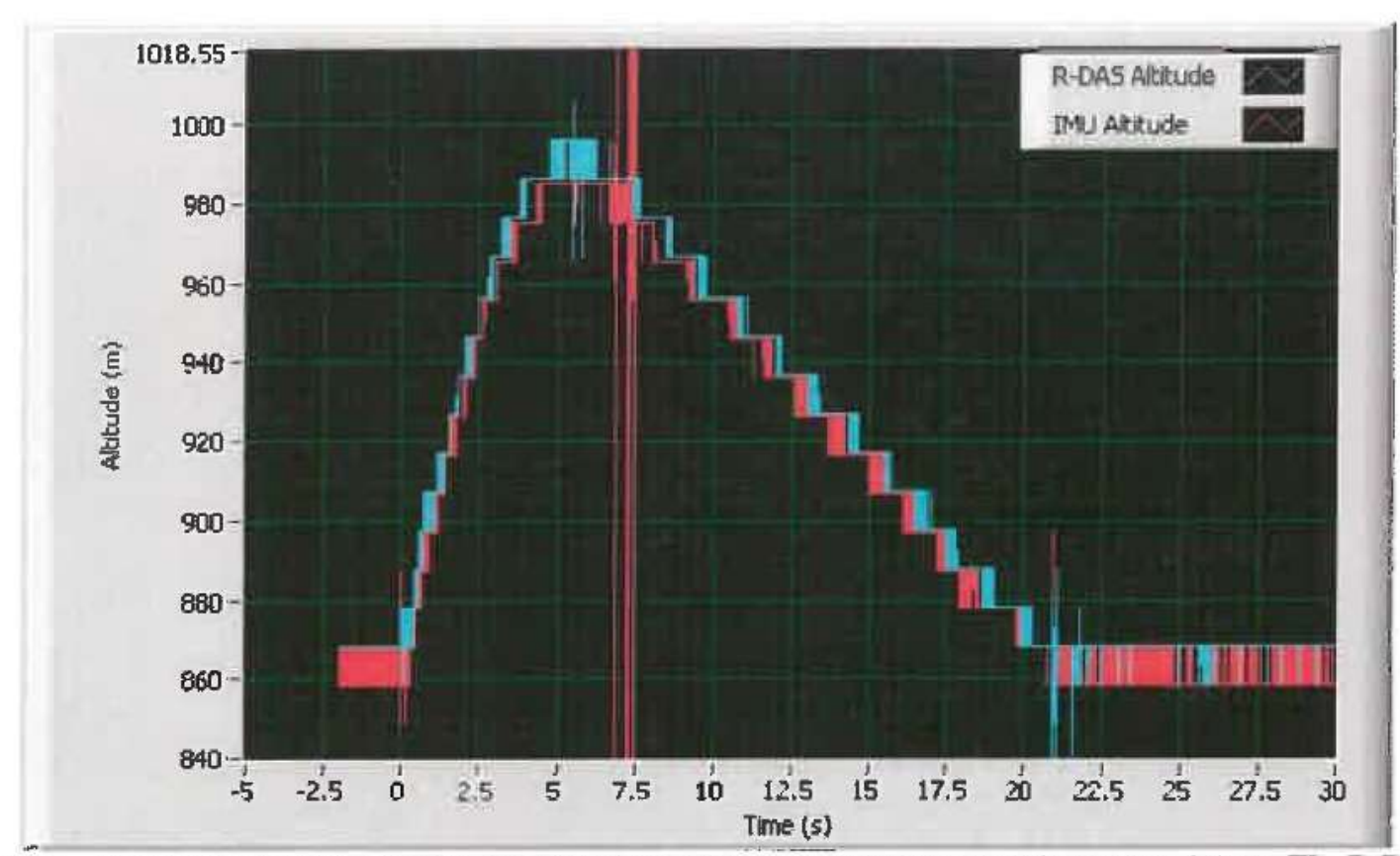

Figure : R-DAS and IMU altitudes, calculated from pressure sensor data, as functions of time. The R-DAS and IMU apogees are $128.16 \mathrm{~m}$ and $118.25 \mathrm{~m}$, respectively.

Figure 5. Rocket altitude calculated from R-DAS tiny pressure sensors in 2008

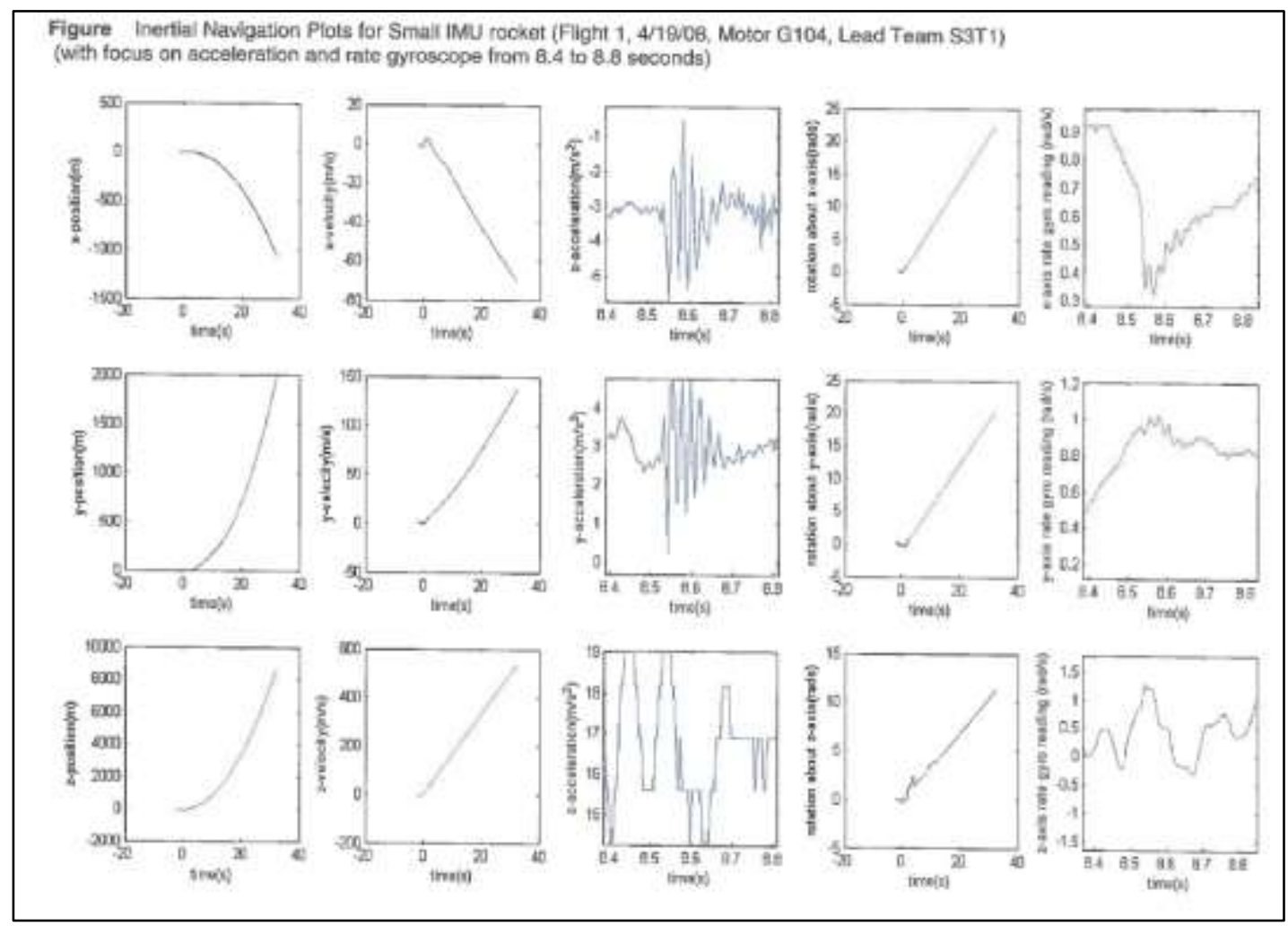

Figure 6. Student work showing IMU raw data from R-DAS tiny and calculated vehicle trajectory $-\mathbf{2 0 0 8}$ 


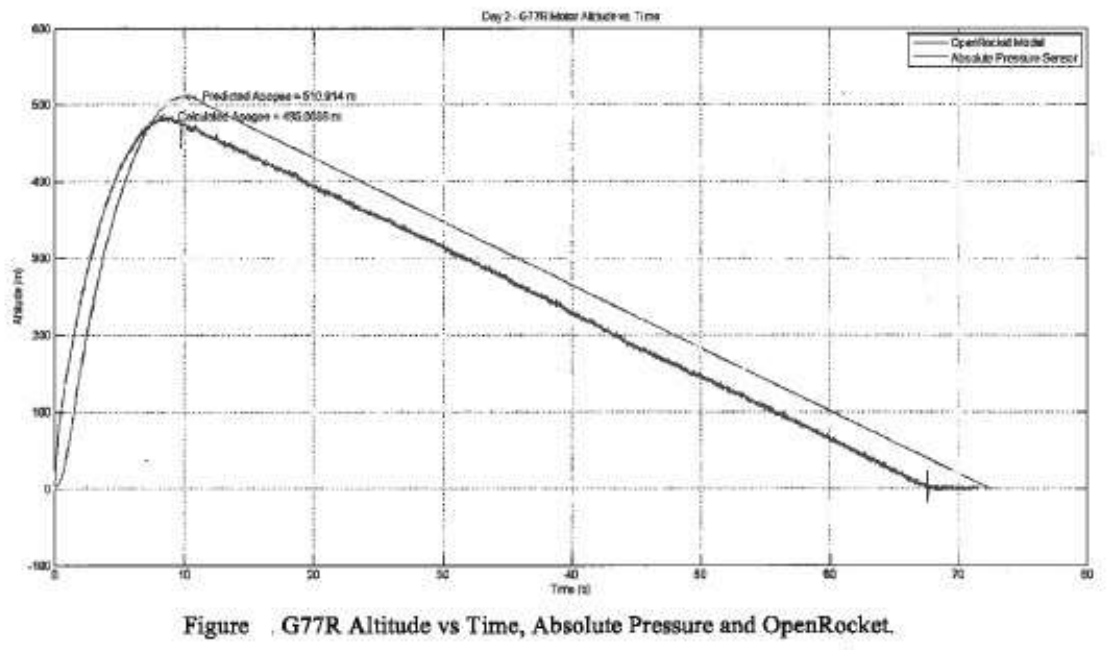

Figure 7. Altitude versus time from absolute pressure sensor data taking with MuddLog16 v4 -- 2015

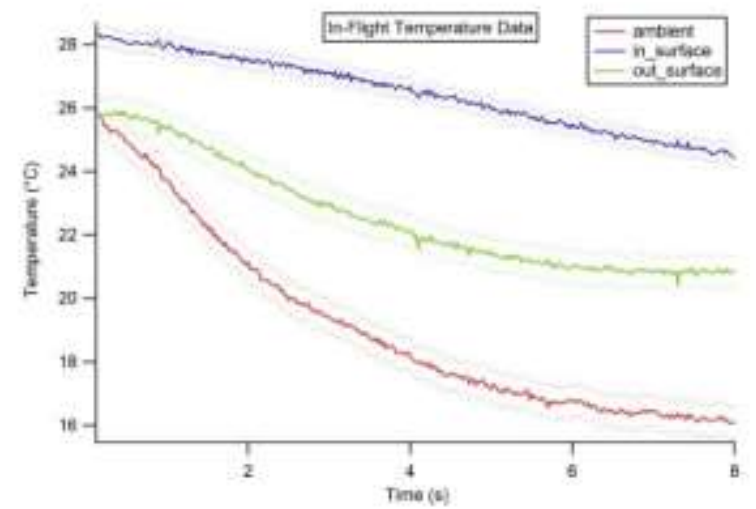

Figure : The temperature measured by the thermocouples on the inner and outer surface and outside of the rocket during ascent.

Figure 8. Thermocouple data taken using MuddLog16 v3 -- 2014

\section{Results}

We assessed learning objectives using student work from multiple years of E80. The low-level objectives of Table 1 were assessed using anecdotal evidence based on student performance in lab sessions, and on outcomes from rocket flights. We also assessed the objectives from Table 5 based on students' final technical report on the rocket flights and analysis of the flight data. We assessed reports from 2008, which was the Beta Version of the course and the R-DAS tiny was 
used as the data logger; 2013 and 2014, during which time v3 of the MuddLog16 data logger was used; and 2015, when both v3 and v4 of MuddLog16 were employed.

The first set of assessments was to measure the degree to which the students were able to focus on the student list (Table 1) and ignore the behind the scenes list. As part of the students' preparation for the field experience (the rocket flights), instructors check-off on various aspects of the students' work. These include sensor selection, power and conditioning circuit design, and rationale for these choices. Each instructor considered the low-level objectives presented in Table 1 (The Student List), and noted the following results.

Regarding the objective, "Scale the input voltages to the proper input range of for the data logger," the results were essentially identical for the pre-MuddLog16 and MuddLog16 teams. Both groups designed circuits to properly scale the sensor outputs to the correct range for the data logger. The custom design did not affect student performance. Student performance on the objective, "Condition the signal to account for the data logger input impedance," was improved by introduction of the custom data logger. The pre-MuddLog16students ignored the input impedance much more frequently than the MuddLog16students. The MuddLog16definitely improved the student consideration of buffering inputs.

The next two objectives in Table 1 pertain to the number of channels and the sampling rate. Students using the R-DAS and Logomatic reported frustration in their inability to compare multiple flight phenomena (for example, rocket velocity compared to vibration or external temperature data.) After the introduction of the MuddLog16, this type of student comment disappeared. Regarding sampling rate, pre-MuddLog16, we noted students made frequent reference to the frustrations of trying to find aliased or folded modal vibrations in the data, and struggling to determine if frequency-response-function peaks corresponded to the correct mode. Once we introduced the custom data logger, with its explicitly-higher sampling rate, students have not needed to analyze aliased/folded data. Students are required to explain their choice of sample rate when choosing sensors and designing circuitry, but due to the availability of high sample rates, very few students need to deal with aliased/folded data.

The last objective on Table 1 relates to powering the data logger using a battery. We saw no difference in student performance as we changed data loggers; however, fewer data loggers were damaged or destroyed due to improper student wiring after we began using the custom data logger.

We also examined student work (final reports) to assess the objectives $1 \mathrm{~B}, 2 \mathrm{~A}$, and $2 \mathrm{C}$ presented in Table 5; rubric assessments were used. Objective 1, "Demonstrate hardware and equipment skills", was split into four sub-objectives describing laboratory equipment skills, field equipment skills, debugging/troubleshooting of experimental set ups, and the design/build/fly of the sensor 
package. Objective 1B, which focuses on the use of data acquisition systems, was used to assess student learning associated with use of the data logger. Objective 2, "Demonstrate experimental and analytical skills", was sub-divided into the three objectives concerning the planning of experiments to answer open-ended questions; the manipulation and presentation of experimentally-obtained data to answer such questions; and the comparison of experimentallyobtained data to expected values. Objectives $2 \mathrm{~A}$ and $2 \mathrm{C}$ were assessed.

Tables 6 through 8 present the rubrics used to assess the student work. A 5-point scale was used, with 5 being the highest score. For example, Table 6 , which is the rubric used to assess the safe and proper use of data acquisition systems, defines a score of 5 as a final report which includes conditioning circuit schematics with values; explains that the data logger inputs are from 0 to $3.3 \mathrm{~V}$; includes information on chosen sample rates; describes how signal aliasing was avoided or used; and lists rocket modifications necessary for sensor functioning. A score of 4 was assigned to reports that were missing one of those items, and so on down to a score of 1 which had none of these descriptions.

\section{Table 5. Description of Assessed Course Objectives}

\begin{tabular}{|l|l|}
\hline Objective 1 & Demonstrate hardware and equipment skills \\
\hline 1B & $\begin{array}{l}\text { Demonstrate the safe and proper use of computer-based and embedded- } \\
\text { processor-based data acquisition systems. This includes explaining the } \\
\text { circuitry to condition the expected sensor output signal into the 0-to-3.3V } \\
\text { @ 2k-ohm input range of the data logger. Description of sample rates and } \\
\text { how these were chosen to avoid or use aliasing should be included. } \\
\text { Explanations of rocket modifications to ensure proper functioning of } \\
\text { sensors should be included. }\end{array}$ \\
\hline Objective 2 & Demonstrate experimental and analytical skills \\
\hline 2A & $\begin{array}{l}\text { Demonstrate the design/planning and completion of safe experiments to } \\
\text { answer open-ended questions }\end{array}$ \\
\hline 2C & $\begin{array}{l}\text { Analyze and compare the results of mathematical and computer modeling } \\
\text { of an experiment with actual experimental results }\end{array}$ \\
\hline
\end{tabular}


Table 6. Rubric for Objective 1B: Safe and proper use of data acquisition systems

\begin{tabular}{|l|l|}
\hline Score & Rubric \\
\hline 5 & $\begin{array}{l}\text { Included circuit schematics with values; explained } 0 \text { to } 3.3 \mathrm{~V} \text {; included } \\
\text { information on chosen sample rates; how to avoid/use aliasing was addressed; } \\
\text { rocket modifications for sensor functioning were described }\end{array}$ \\
\hline 4 & The description was missing one of these \\
\hline 3 & The description was missing two of these \\
\hline 2 & The description was missing many \\
\hline 1 & No description of any of these \\
\hline
\end{tabular}

Table 7. Rubric for Objective 2A: Completion of experiments to answer open-ended questions

\begin{tabular}{|l|l|}
\hline Score & Rubric \\
\hline 5 & Got meaningful data on every sensor \\
\hline 4 & Got data on all sensors but some of the data were not good in some way \\
\hline 3 & Got some meaningful data on some of the sensors - more than half \\
\hline 2 & Got meaningful data on one sensor \\
\hline 1 & No data \\
\hline
\end{tabular}

Table 9 presents the results of the assessment of the final reports. Objective 1B, which assessed the use and understanding of the data logger, showed 2015 with the highest score of 4.1, when MuddLog16 v3 and v4 were used. Interestingly, our Beta Version in 2008 using the R-DAS tiny had a not unreasonable score of 3.2. The R-DAS used in 2008 had a maximum sampling rate of $200 \mathrm{~Hz}$, and the necessity of analyzing higher-frequency vibration data required that students explicitly dealt with aliased data. This was apparent in the final reports, where students did a good job of describing the sampling rate of the R-DAS, and the aliasing in their vibration data. In contrast, students who used the MuddLog16 (2013, 2014, and 2015) often glossed over sampling rate and aliasing in their final report, with no mention of the frequency content of the sensors signals. Assessment of 1B also showed that students using the MuddLog16 scored higher in terms of demonstrating understanding and design of conditioning circuitry. This makes sense since the students were required to design such circuitry. Students in 2008, where the conditioning circuitry were already designed and provided, did not score as highly. 
Table 8. Rubric for Objective 2C: Analysis/comparison of experimental and expected results

\begin{tabular}{|l|l|}
\hline Score & Rubric \\
\hline 5 & $\begin{array}{l}\text { Compared model/expected and experimental data both graphically/tabularly and } \\
\text { in the text. Described well. }\end{array}$ \\
\hline 4 & $\begin{array}{l}\text { Adequate analysis/comparison. Possibly missing some information or described } \\
\text { in an OK fashion }\end{array}$ \\
\hline 3 & $\begin{array}{l}\text { Took a swing at making a comparison, possibly missing graphical/tabular } \\
\text { comparison or average description of comparison }\end{array}$ \\
\hline 2 & Not adequate. Missing more information/graphs/tables/comparisons \\
\hline 1 & No comparison, no analysis \\
\hline
\end{tabular}

Table 9. Rubric assessment results.

\begin{tabular}{|l|l|l|l|l|}
\hline & $\begin{array}{l}\text { R-DAS tiny } \\
2008(\mathrm{n}=5)\end{array}$ & $\begin{array}{l}\text { MuddLog v16 } \\
2013(\mathrm{n}=3)\end{array}$ & $\begin{array}{l}\text { MuddLog v16 } \\
\text { 2014 (n=5) }\end{array}$ & $\begin{array}{l}\text { MuddLog v16 } \\
2015(\mathrm{n}=16)\end{array}$ \\
\hline $\begin{array}{l}\text { 1B } \\
\text { Demonstrate } \\
\text { Data } \\
\text { Acquisition }\end{array}$ & 3.2 & 2.8 & 3.6 & 4.1 \\
\hline $\begin{array}{l}\text { 2A Design \& } \\
\text { Complete } \\
\text { Experiment }\end{array}$ & 2.9 & 4.3 & 4 & 3.8 \\
\hline $\begin{array}{l}\text { 2C Compare } \\
\text { Modeling with } \\
\text { Experiment }\end{array}$ & 3.5 & 4.3 & 3.7 & 2.6 \\
\hline
\end{tabular}

The acquisition of experimental data using the logger (Objective 2A) was affected by many things. These included rocket motor issues; data logger problems; and parachute deployment problems. In 2008, some of the rockets were destroyed during flight in Beta testing due to design issues with these vehicles. For many of those incidents, the data from the R-DAS data logger were completely lost or were corrupted. V3 of the MuddLog 16 featured a SD card holder that sometimes 'allowed' the card to pop out during flight, causing loss of all data. This issue came to light in 2013. Fortunately, most teams were able to get in four flights over two launch days, and 
a quick fix using tape to secure the card was generally successful in preventing the malfunction. In 2014, a lab assistant mistakenly assembled some of the motors incorrectly, leading to flight failures where data were either not acquired, or were not useful in answering their scientific questions. This unfortunate occurrence was exacerbated by the students only being able to get one day of launches in, as the second launch was cancelled due to bad weather. In 2015, the data logger and vehicles performed well, but day 2 launches featured very-high winds, which led to issues with rocket (and data logger) recovery.

The assessments show that the 2008 course (with the R-DAS data logger and vehicle destruction) with the lowest score for 2A, with a value of 2.9. Years 2013 through 2015, where the MuddLog 16 was employed, had much higher scores of 4.3, 4, and 3.8. The assessments show that the acquisition of experimental data was much improved due to the use of the MuddLog16 data logger.

Objective 2C was assessed to determine how well students compared their experimentallyobtained data with expected results. This assessment shows that students don't always present their comparisons in the best way, with the majority of scores at around 3.6 out of 5. Most commonly, students did not use graphs or tables to clearly and efficiently to compare data. Previous assessments ${ }^{14}$ also showed that E80 students did not generally present overlaid data on plots; side-by-side plots were sometimes presented, even though the students were capable of producing more-sophisticated plots. Tabular comparisons were more commonly seen in the final reports, especially when students reported measured and predicted apogee altitude. It is difficult to point to the change in data logger from the R-DAS to the MuddLog16 as affecting student performance for this objective - changes in our college core physics lab may have had more of an effect here- however, given that the data quality is so much better with the MuddLog16, it was disappointing that students did not take advantage of such beautiful data in presenting their results.

Other notable characteristics from a comparison of the data in student final presentations from 2008 with those from 2013 through 2015 (without rubric assessment) show that any single data set from 2008 only involved one type of sensor, either IMU, vibration, or temperature and pressure, whereas the 2013 through 2015 data usually involved multiple sensor types with the students pointing out correlations between flight events (liftoff, parachute deployment, etc.) with changes in the data in multiple sensor types.

In summary, the quality of the data the students presented in 2013 through 2015 were vastly improved over the 2008 R-DAS data in terms of number of sensors/channels, resolution, and avoiding problems with aliasing. The later students spent much less time worrying about aliasing and more time focused on their experimental objectives. The student learning assessment showed marked improvement in learning objectives $1 \mathrm{~B}$ and $2 \mathrm{~A}$ but essentially no change in $2 \mathrm{C}$. The new data loggers changed the focus of the students from the limitations of their data acquisition to the 
limitations of their experiments, consistent with the goals for the course. The student reports are consistent with all of the behind-the-scenes list of design objectives being achieved. The new hardware largely achieved its design objectives and showed improvement in some, but not all, student learning objectives.

\section{Conclusions}

The quality of data the students acquired using the MuddLog16 has improved dramatically, especially compared to data taken with the R-DAS in 2008. Rubric assessments showed that the students improved skills in the safe and proper use of computer-based and embedded-processorbased data acquisition systems, and in the design/planning and completion of safe experiments to answer open-ended questions. Using the new data logger, students measured data from multiple sensor types at higher resolution (compared to the previous loggers,) allowing more sophisticated analysis of flight events and the scientific phenomena that the students were measuring. The focus of the students changed as limitations of the various data loggers were addressed in the design of the MuddLog16. Students were able to focus on their experiments, acquiring data from multiple sensors with sufficient resolution to address their scientific goals, which satisfied learning objectives for the course.

However, although one of our objectives was to design a data logger with a high enough sampling rate so that aliasing of vibration data was avoided (thus allowing students to focus on other learning objectives), the implementation of just such a design may have been detrimental to other aspects of student learning. As mentioned earlier, due to the difficulties of delivering an intense course to sophomore students, we made choices regarding the ranking of our learning objectives. In future offerings of the course, it may be useful to design a short lab experiment that requires the students to explicitly acquire and analyze aliased data. For example, we could provide the students with a data logger with a sample rate similar to the R-DAS (a few hundred SPS) to analyze vibration modes of a cantilever beam. This could be done in the first half of the course, before the students start using the custom data logger with higher sampling rate, thus allowing students to compare differences between data quality of the various data loggers.

At the time E80 was conceived, we wanted a way for students to fly a full complement of analog sensors on an inexpensive rocket. Data acquisition systems that could meet the desired performance specs were far too large and expensive. The concept of small embedded systems such as the Arduino were just coming onto the scene. Since then, whole families of inexpensive embeddable computers have appeared, from the Arduino, to the Raspberry Pi, to Gumstix. Harvey Mudd has developed both the Mudduino, used in the 1st-year autonomous vehicles course, and the MuddLogg16, used in the sophomore engineering laboratory. Both required a confluence of professors who understood the design objectives and students willing to do the design work. The MuddLogg costs about three time the purchase price of a Raspberry Pi, but it 
will fit in a 1.8-inch rocket payload tube and acquire 16 channels of 16-bit data at $400 \mathrm{kSPS}$. The trade-off between designing and producing your own board, and adapting existing commercial and hobbyist hardware is very case specific, but either path is much less expensive than it used to be. For the rockets in E80 the custom design has been worth it. However, were we starting from scratch today, we would probably go with mostly digital sensors and interface them with one of the commercial embeddable systems. It is now possible for all students to have access to inexpensive hardware that has capabilities that weren't available for any price 20 year ago.

\section{Bibliographic Information}

1) Biswajit Ray, 'An Instrumentation and Data Acquisition Course for Electronics Engineering Technology Students', Proceedings of the 2003 American Society for Engineering Education Annual Conference \& Exposition 2) Biswajit Ray, 'Instrumentation and Data Acquisition Projects by Sophomore-Level EET Students', Proceedings of the 2004 American Society for Engineering Education Annual Conference \& Exposition

3) John L. Schroeder and Christopher C. Weiss, 2008: Integrating Research and Education through Measurement and Analysis. Bull. Amer. Meteor. Soc., 89, 793-798.

4) Greg Mason, 'A handheld data acquisition system for use in an undergraduate data acquisition course', IEEE Transactions On Education, Vol. 45, No. 4, November 2002

5) Steve Warren, Xiongjie Dong, Tim J. Sobering, Jason Yao, 'A Rapid Analysis and Signal Conditioning Laboratory (RASCL) Design Compatible with the National Instruments myDAQ ${ }^{\circledR}$ Platform’, American Society for Engineering Education, 2011

6) “Telemetry", AED Electronics, http://www.aedelectronics.nl/rdas/telemetry.htm, accessed 1/13/2016

7) "R-DAS tiny", AED Electronics, http://www.aedelectronics.nl/rdas/tiny.htm, accessed 1/13/2016

8) "R-DAS PCB Layout", AED Electronics, http://www.aedelectronics.nl/rdas/images/rdas tiny.jpg, accessed $1 / 29 / 2016$

9) “SparkFun Logomatic”, SparkFun, https://cdn.sparkfun.com//assets/parts/1/7/2/8/08627-04.jpg, accessed $1 / 29 / 2016$

10) “MuddLog16 v3 Layout”, HMC E80, http://www.eng.hmc.edu/NewE80/PDFs/MuddLog16Layout.pdf, accessed 1/13/2016

11) “MuddLog16 v3 Schematic”, HMC E80, http://www.eng.hmc.edu/NewE80/PDFs/MuddLog16Schematic.pdf, accessed 1/13/2016

12) “MuddLog v4 Layout”, HMC E80, http://www.eng.hmc.edu/NewE80/PDFs/MuddLogg16_v4_Layout.pdf, accessed 1/13/2016

13) “MuddLog v4 Schematic”, HMC E80, http://www.eng.hmc.edu/NewE80/PDFs/MuddLogg16_v4_Scheme.pdf, accessed 1/13/2016

14) Mary Cardenas, "An Implementation of Electronic Laboratory Notebooks (ELN) Using a Learning Management System Platform in an Undergraduate Experimental Engineering Course", Proceedings of the 2014 American Society for Engineering Education Annual Conference and Exposition, 2014.

Acknowledgements: We would like to thank Tyler Smelt (HMC '13) for his invaluable help in designing the MuddLog16. 